\title{
An Oversampled Channelized UWB Receiver with Transmitted Reference Modulation
}

\author{
Lei Feng, Student Member, IEEE, Won Namgoong, Member, IEEE
}

\begin{abstract}
To digitize the ultra-wideband (UWB) signal at its Nyquist rate, a frequency channelized receiver for UWB radio based on hybrid filter banks (HFB) is presented. Among the challenges of such receivers are the uncertainties of the analog analysis filters and the slow convergence speed. To overcome these problems, a channelized receiver operating at slightly above the critically sampling rate is presented. The proposed receiver, which is designed for use in transmitted reference (TR) systems, combines the synthesis filters and the matched filter so that the joint response of the analysis filter and the propagation channel can be estimated independently in each subband. When the input noise is colored or a narrowband interference (NBI) is present, the weighting in each subband can be adaptively adjusted so to approximate the noise whitening and matched filtering operation for near optimal detection. The adaptive performance of the proposed receiver is slightly better than an ideal fullband receiver when the input noise is white and significantly better when a NBI is present. The effect of the automatic gain controller (AGC) and the analog-to-digital converter (ADC) are also considered. The proposed receiver with 3-bit ADC seems sufficient to achieve performance comparable to an infinite bit channelized receiver even in the presence of large NBI.
\end{abstract}

Index Terms-Ultra-wideband, transmitted reference, channelized receiver, hybrid filter bank, analog-to-digital converter, narrowband interference.

\section{INTRODUCTION}

A MAJOR challenge in ultra-wideband (UWB) communication is achieving accurate channel estimation. Transmitted reference (TR) modulation schemes have been proposed because of the ease of channel estimation in a dense multipath environment [1]. In the standard TR system, a reference pulse is transmitted before each data-modulated pulse to simplify the estimation of the multipath channel response. At the receiver, the transmitted data is detected by delaying the received reference pulse then correlating with the data-modulated pulse.

Although the UWB TR receiver structure with a delay element seems simple at first glance, an integrated low-power receiver is difficult to implement in practice. The primary challenge is in realizing the extremely wideband analog delay element that is used in TR systems. A continuous-time wideband delay element requires a filter response whose group delay is constant over the frequency band of interest, which can be in excess of a gigahertz in UWB systems. Such circuits are difficult to implement, especially considering the large delay (and fine resolution) that would be required in TR

L. Feng and W. Namgoong are with the University of Southern California. Email: leifeng@usc.edu, namgoong@usc.edu.

This work was supported in part by the Army Research Office under contract number DAAD19-01-1-0477 and National Science Foundation under contract number ECS-0134629. systems. Furthermore, improved TR systems such as [2][3] require additional delay capability, making an integrated UWB TR receiver even more difficult.

To avoid the use of an analog delay element and to achieve high performance, the UWB TR receiver needs to digitize the UWB signal at least at the signal Nyquist rate (usually several gigahertz) so that all of the receiver functions can be performed digitally. Digital operations can be performed very efficiently in modern scaled CMOS technology. The primary implementation challenge is designing the high-speed ADC. Since designing a single ADC to operate at the required sampling frequencies (of, for example, several GSamples/sec) is difficult, parallel ADC architectures with each ADC operating at a fraction of the effective sampling frequency need to be employed [4][5].

To sample at a fraction of the effective sampling frequency, a frequency channelized receiver based on hybrid filter banks (HFB) (i.e., continuous-time analysis filters and discrete-time synthesis filters) has been proposed in [6]. Among the advantages of the frequency channelized receiver compared to the more conventional time channelized (i.e., time-interleaved ADC) receivers are the ease of designing the sample/hold circuitries, greater robustness to jitter/phase noise, and reduced ADC dynamic range requirements [6].

In the frequency channelized receiver, signal detection can be achieved by first reconstructing the channelized signal then processing the sampled full-band signal as in the conventional receiver. Design of perfect reconstruction (PR) or approximately PR HFBs have been explored [7][8]. A potential problem with this approach is that designing the digital synthesis filters requires accurate knowledge of the transfer functions of the analog analysis filters, which may be unavailable in practice because of the variations resulting from temperature and fabrication process uncertainties.

The distortion caused by the propagation channel and the analysis filters can be compensated by employing adaptive synthesis filters to recover the transmitted signals. The primary drawback is the slow convergence speed as shown in [9] and [10]. Oversampled filter banks have also been proposed to achieve faster convergence [11]. The convergence speed, however, is still not sufficiently fast in time-varying UWB channels.

In this paper, we propose an oversampled channelized UWB receiver that outperforms the ideal full-band TR receiver, especially in the presence of narrowband interference (NBI). Instead of determining the synthesis filters that reconstruct the received full-band signal waveform, which suffers from slow convergence, the proposed receiver performs signal detection 
by estimating the combined responses of the analysis filter and the propagation channel. If the analysis filters are designed to satisfy the power complementary condition, which can be achieved as described in a later section, the PR synthesis filters are the time-reversed complex conjugates of the analysis filters [12]. Since the matched filter is also the time-reversed complex conjugate of the propagation channel, the matched filter and the synthesis filter can be combined and the estimate of the pulse response for detection can be obtained in a TR system by simply averaging the reference pulses in each of the subband channels, assuming that the pulse spacing is larger than the delay spread. Analysis shows that the oversampled channelized receiver outperforms the full-band receiver when the additive noise is white.

In addition to the additive white noise, the proposed oversampled channelized receiver effectively handles colored noise and NBI unlike in the standard full-band TR receiver. When the additive noise is colored or a NBI is present, optimal detection is achieved by noise whitening then matched filtering the received signal. The proposed oversampled channelized receiver can be made to adaptively approximate the optimal detection process by appropriately combining the subband correlation values. The detection process also considers the effect of the AGCs, which are applied in each subband channel in practice so that the full dynamic range of each ADC is used. The proposed channelized receiver achieves significantly higher performance than a full-band receiver because of its ability to isolate the effects of the NBI.

This paper is organized as follows. Section II introduces the system model. Section III presents the proposed receiver structure based on the PR filter bank. In Section IV, the estimation and detection performance is analyzed. AGC, ADC and NBI effects are considered in Section V. Simulation results are provided in Section VI and conclusions are drawn in Section VII.

\section{SYSTEM MODEL}

The UWB signal is a time-hopping pulse train. To simplify the problem, we assume that the pulses are transmitted in fixed time intervals and that the interval is sufficiently large to prevent interference between consecutive pulses. Assuming a block of $N_{b}$ pulses are transmitted, the received UWB signal $r(t)$ is

$$
r(t)=\sum_{k=0}^{N_{b}-1} a_{k} s(t-k T)+v(t)
$$

where $a_{k}$ is the $k$ th transmitted antipodal symbol, $T$ is the time interval between consecutive pulses, $s(t)$ is the received signal pulse response, and $v(t)$ is the additive white Gaussian noise (AWGN). In each transmission block, we assume that the first $N_{r}$ pulses are reference pulses and are not data modulated, i.e., $a_{k}=1$ for $0 \leq k<N_{r}$. The remaining pulses are data pulses.

Optimal receivers for TR systems based on the generalized likelihood ratio test are described in [2][13]. Since optimal receivers suffer from high complexity, we only consider the crosscorrelation TR receivers. In particular, we focus on the

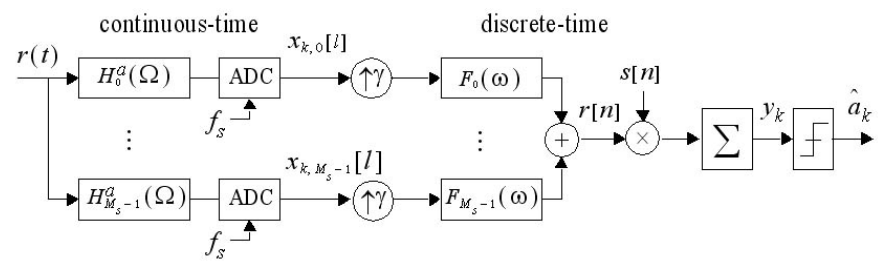

Fig. 1. A frequency channelized receiver with a PR filter bank.

averaged TR receiver [2], which operates by averaging the $N_{r}$ reference pulses to improve the pulse response estimate, and then correlate this estimate with data pulses for detection. The averaged TR receiver is shown in [2] to achieve performance comparable to the optimal TR receiver.

The analog front-end of the frequency channelized receiver with a PR filter bank is shown in Fig. 1. The received signal $r(t)$ is passed through a bank of continuous-time analysis filters $\left\{H_{m}^{a}(\Omega)\right\}$ with $m \in\left\{0,1, \ldots, M_{s}-1\right\}$, sampled and digitized by the ADC at a rate of $1 / T_{s}$, upsampled by $\gamma$, filtered by the synthesis filters $\left\{F_{m}(\omega)\right\}$, then summed to reconstruct the received signal. The effective sampling frequency of the $\mathrm{HFB}$ is $1 / T_{e}$ and is related to the ADC sampling frequency $1 / T_{s}$ by $\gamma$ (i.e., $T_{e}=T_{s} / \gamma$ ) and to the pulse interval $T$ by $N$ (i.e., $\left.T_{e}=T / N\right)$.

Assuming that $\left\{H_{m}^{a}(\Omega)\right\}$ and $\left\{F_{m}(\omega)\right\}$ achieve PR, the reconstructed signal $r[n]$ is ideally correlated with the sampled pulse response $s[n]\left(=s\left(n T_{e}\right)\right)$ then passed through a slicer for detection. In the TR system, an estimate of $s[n]$ is used for correlation and is obtained by averaging the reference pulses. Although the PR assumption is made for deriving the HFB receiver, the $\mathrm{PR}$ filters are not implemented as described in the following section.

The $l$ th sample of the $m$ th subband channel in the $k$ th pulse is

$$
x_{k, m}[l]=\int_{-\infty}^{\infty} h_{m}^{a}\left(k T+l T_{s}-\tau\right) r(\tau) d \tau
$$

where $h_{m}^{a}(t)$ is the impulse response of the $m$ th analysis filter $H_{m}^{a}(\Omega)$. To obtain the discrete equivalent model of the frequency channelized receiver in Fig. 1, which is subsequently used for simulation and analysis, the received signal $r(t)$ is sampled at $\kappa / T_{e}$, where $\kappa$ is a positive integer. Since $\left\{H_{m}^{a}(\Omega)\right\}$ and $r(t)$ are bandlimited, the discrete model is accurate for sufficiently large $\kappa$. Although $\kappa=4$ is used when simulating for accuracy, we assume $\kappa=1$ for simplicity when analyzing the frequency channelized receiver. This is a reasonable assumption since $\left\{H_{m}^{a}(\Omega)\right\}$ and $r(t)$ are approximately bandlimited within $1 /\left(2 T_{e}\right)$. Applying the equivalence theorem of digital and analog signal processing [14], (2) becomes

$$
\begin{aligned}
x_{k, m}[l] & =T_{e} \sum_{n=-\infty}^{\infty} h_{m}^{a}\left(k T+l T_{s}-n T_{e}\right) r\left(n T_{e}\right) \\
& =\sum_{n=-\infty}^{\infty} h_{m}[k N+l \gamma-n] r[n]
\end{aligned}
$$


where $h_{m}[n]=T_{e} h_{m}^{a}\left(n T_{e}\right)$ and

$$
r[n]=\sum_{k=0}^{N_{b}-1} a_{k} s\left(n T_{e}-k T\right)+v[n]
$$

In (4), $v[n]$ is the AWGN with a power spectrum density (PSD) that corresponds to that of $v(t)$.

Fig. 2 shows the equivalent discrete model of Fig. 1 with the correlation operation pushed to before the subband summation operation. In the equivalent model, the continuoustime analysis filter $H_{m}^{a}(\Omega)$ is replaced by a discrete-time filter $H_{m}(\omega)$, whose impulse response is $h_{m}[n]$. To form a complete representation of the received signal, $M=2 M_{s}-1$ subbands between 0 and $2 \pi$ are needed because the discrete spectrum covered by the $M_{s}$ subbands is mainly between 0 and $\pi$. Since the received signal is assumed to be real, the remaining spectrum is covered by conjugating signals in the 1 st to $\left(M_{s}-1\right)$ th subbands. We subsequently refer to these subbands as virtual subbands as they are not actually implemented but are used to simplify the analysis. For subbands $m=M_{s}, \ldots, 2 M_{s}-2$, the conjugate samples and the corresponding analysis filters are

$$
\begin{aligned}
x_{m}[l] & =x_{M-m}^{*}[l] \\
h_{m}[n] & =h_{M-m}^{*}[n]
\end{aligned}
$$

We will subsequently use $M$ subbands instead of $M_{s}$ subbands to be consistent with discrete filter bank systems.

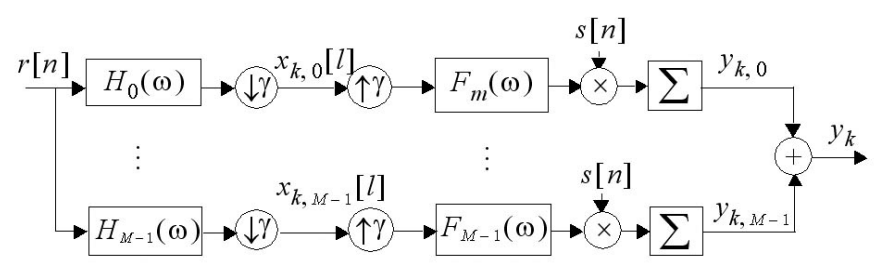

Fig. 2. The discrete equivalent model of the frequency channelized receiver given in Fig. 1 with the correlation operation pushed to before the subband summation operation.

Substituting $r[n]$ in (3) with (4), the ADC output in the $m$ th subband channel of the $k$ th pulse can be written as

$$
x_{k, m}[l]=a_{k} s_{m}[l]+v_{k, m}[l]
$$

where

$$
\begin{gathered}
s_{m}[l]=\sum_{n} h_{m}[\gamma l-n] s[n] \\
v_{k, m}[l]=\sum_{n} h_{m}[\gamma l-n] v[n+k N]
\end{gathered}
$$

After some straightforward mathematical manipulations, the subband correlation output $y_{k, m}$ can be shown to be

$$
y_{k, m}=\sum_{l} x_{k, m}[l]\left(\sum_{n} f_{m}[n-\gamma l] s[n]\right)
$$

where $f_{m}[n]$ is the impulse response of $F_{m}(\omega)$. The input to the slicer is the summation of all the outputs of the equivalent subband channels, i.e.,

$$
y_{k}=\sum_{m=0}^{M-1} y_{k, m}
$$

(10) and (11) suggest that detection in a frequency channelized receiver can be achieved at the ADC sampling rate by appropriately correlating the ADC output then summing. Since no upsampling operation is needed after the ADC, all of the receiver functions are performed at the ADC sampling rate. The reduced operating frequency in the proposed receiver, therefore, relaxes the requirements on the digital circuitries.

\section{THE Proposed RECEIVER}

TR detection in the HFB receiver described in the previous section assumes that the PR conditions are satisfied. The PR conditions for $\left\{F_{m}(\omega)\right\}$ and $\left\{H_{m}(\omega)\right\}$ are:

$$
\begin{gathered}
\sum_{m=0}^{M-1} H_{m}(\omega) F_{m}(\omega)=\gamma \\
\sum_{m=0}^{M-1} H_{m}(\omega-2 \pi \beta / \gamma) F_{m}(\omega)=0
\end{gathered}
$$

for $\beta=1, \ldots, \gamma-1$ and all $\omega$. In practice, satisfying the PR condition is difficult because of the uncertainties in the transfer function of the analog analysis filter $H_{m}(\omega)$. In this section, we describe how detection can be achieved in the HFB receiver for TR systems.

\section{A. TR Detection and signal estimation}

If $\left\{F_{m}(\omega)\right\}$ is designed to be

$$
f_{m}[n]=h_{m}^{*}[-n]
$$

the PR conditions then become

$$
\begin{gathered}
\sum_{m=0}^{M-1}\left|H_{m}(\omega)\right|^{2}=\gamma \\
\sum_{m=0}^{M-1} H_{m}(\omega-2 \pi \beta / \gamma) H_{m}^{*}(\omega)=0
\end{gathered}
$$

The first PR condition given in (15) can be satisfied by designing to be power complementary. The second PR condition given in (16) can be satisfied by operating the HFB slightly above the critically sampling rate. The method for achieving both of these conditions is described in the following subsection. By designing $F_{m}(\omega)$ as in (14), the subband correlation output $y_{k, m}$ given in (10) becomes

$$
y_{k, m}=\sum_{l}\left(a_{k} s_{m}[l]+v_{k, m}[l]\right) s_{m}^{*}[l]
$$

From (17), the advantage of designing $F_{m}(\omega)$ as in (14) becomes clear. The task of determining the correlator (for the averaged TR system) is to simply estimate the subband 
pulse response $s_{m}[l]$ based on the corresponding ADC samples $x_{k, m}[l]$. This estimation can be readily obtained in TR systems by simply averaging the $N_{r}$ reference pulses, i.e., $\hat{s}_{m}[l]=$ $\frac{1}{N_{r}} \sum_{k=0}^{N_{r}-1} x_{k, m}[l]$.

A more intuitive explanation of the estimation and detection approach described above can be given as follows. If the analysis filters are designed to satisfy the power complementary condition while being sufficiently oversampled to satisfy the aliasing condition in (16), the PR synthesis filters are the time-reversed complex conjugates of the analysis filters [12]. Since the matched filter is also the time-reversed complex conjugate of the propagation channel, the matched filter and the synthesis filter can be combined and the estimate of the pulse response for detection can be obtained by simply averaging the combined responses of the propagation channel and the analysis filters.

\section{B. Subband filters}

Instead of using a bank of bandpass filters for $\left\{H_{m}(\omega)\right\}$, the frequency channelization can be achieved by employing a bank of complex mixers and lowpass filters [12]. The advantages of this channelization scheme are two-fold: the design of sharp bandpass filters centered at high frequencies, which are difficult to realize, is avoided; the ADC sample/hold circuitries are relaxed, since the ADC only sees the bandwidth of the lowpass filter.

The $m$ th subband channel employing such channelization approach is shown in Fig. 3(a). The mixer downconversion frequency of the $m$ th subband channel is $m f_{a}$, and the lowpass filter, which is the same for all subband channels, is $G(\omega)$. To remove the dependency on the analog filter $G(\omega)$, a digital filter $W(\omega)$ is introduced. The effect of $W(\omega)$ on the transfer function of the analysis filter is best understood by pushing to before the decimator and pushing the mixer to after the filters as shown in Fig. 3(b). In this equivalent model, $G_{m}(\omega)=$ $G\left(\omega-m \omega_{a} T_{e}\right)$ and $W_{m}(\omega)=W\left(\gamma \omega-m \omega_{a} T_{e}\right)$, where $\omega_{a}=$ $2 \pi f_{a}$. The analysis filter can now be viewed as being

$$
H_{m}(\omega)=G_{m}(\omega) W_{m}(\omega)
$$

The mixer after the decimator can be absorbed by the correlator.

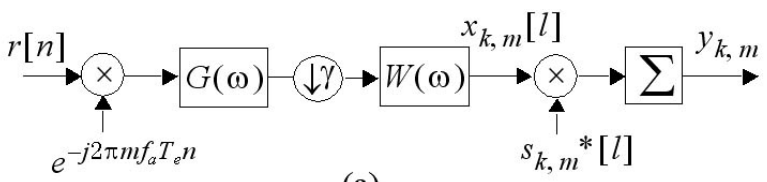

(a)

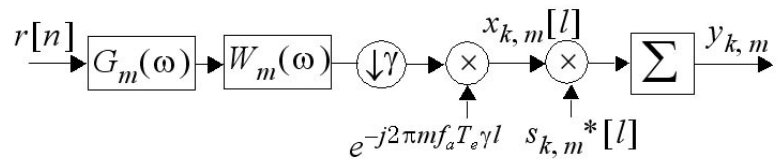

(b)

Fig. 3. (a) Frequency channelization using mixers and lowpass filters; (b) An equivalent model of (a).
$W(\gamma \omega)$ consists of multiple images due to upsampling.

$$
W(\gamma \omega)=\sum_{\nu=0}^{\gamma-1} W^{p}\left(\omega-\frac{2 \pi \nu}{\gamma}\right)
$$

where $W^{p}(\omega)$ is a lowpass filter. Since we are interested in $W^{p}(\omega)$ only, $G(\omega)$ is designed to be sharp enough to eliminate the images as shown in Fig. 4. The analysis filter can now be viewed as being

$$
H_{m}(\omega)=G_{m}(\omega) W^{p}\left(\omega-m \omega_{a} T_{e}\right)
$$

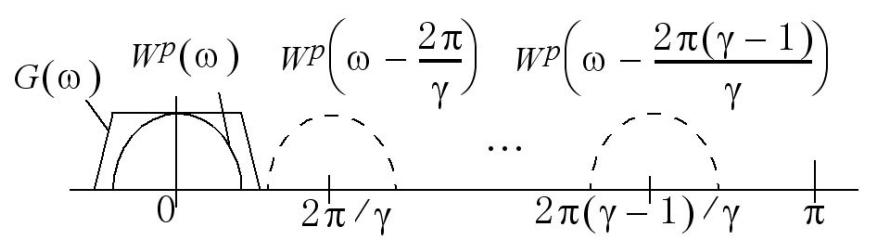

Fig. 4. Filter images in the equivalent model.

To satisfy the second PR condition given in (16), the filter bank should be sufficiently oversampled and $H_{m}(\omega)$ made adequately sharp to remove all of its spectral images. This is equivalent to designing $W^{p}(\omega)$ so that the overlap between its images is negligible. Once $W^{p}(\omega)$ is designed, $W(\omega)$ can be obtained by decimation.

In this channelization approach, the first PR condition given in (12) and (15) is

$$
\sum_{m=0}^{M-1}\left|G_{m}(\omega)\right|^{2}\left|W^{p}\left(\omega-m \omega_{a} T_{e}\right)\right|^{2}=\gamma
$$

To remove the uncertainty in $G_{m}(\omega)$, we require that only the magnitude of $G_{m}(\omega)$ be flat within the passband of $W^{p}\left(\omega-m \omega_{a} T_{e}\right)$. The phase of $G_{m}(\omega)$, which is often difficult to control, can be arbitrary. The PR condition then becomes a function of only the digital filter $W^{p}(\omega)$, which can be accurately controlled, i.e.,

$$
\sum_{m=0}^{M-1}\left|W^{p}\left(\omega-m \omega_{a} T_{e}\right)\right|^{2}=\gamma
$$

\section{The Proposed Channelized Receiver}

The proposed channelized receiver is shown in Fig. 5. As described in the previous subsection, the analysis filters are realized by a bank of equally spaced mixers and identical lowpass filters $G(\omega)$. A digital filter $W(\omega)$ is also included to control the transfer function of the analysis filters. Since the received signal $r(t)$ is real, the filter banks operate only on the positive frequency spectrum except for the zeroth subband channel, which operates on both the positive and the negative frequencies. Signal detection, therefore, is performed after scaling the output of the nonzero subband channels by two and taking the real part of the summation.

The proposed oversampled TR HFB receiver detects the received signal by correlating the ADC output $x_{k, m}[l]$ by $s_{m}^{*}[l]$ 


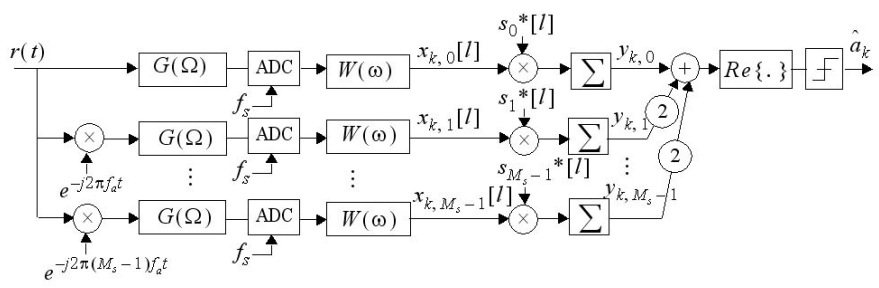

Fig. 5. The proposed UWB receiver.

as described in (17). In practice, however, $s_{m}[l]$ is unknown at the receiver and must be estimated based on $N_{r}$ reference pulses, i.e., $x_{k, m}[l]$ for $0 \leq k<N_{r}$. As stated earlier, the estimation in TR system can be achieved by simply averaging the reference pulses.

\section{Subband Estimation And Detection}

In this section, we compare the performance of a TR HFB receiver with that of a full-band receiver. In the TR system, $N_{r}$ reference pulses at the beginning of each block are used to estimate the pulse response, which is then used to decode the remaining data symbols by correlating the estimate with the data pulses.

\section{A. Full-Band Receiver}

Assuming $N_{s}$ samples are collected at a rate of $1 / T_{e}$ for each pulse, the received $k$ th pulse can be represented in vector form as

$$
\mathbf{r}_{k}=a_{k} \mathbf{s}+\mathbf{v}_{k}
$$

where $\mathbf{v}_{k}$ is zero mean white noise with covariance matrix $\sigma^{2} \mathbf{I}$ and $\mathbf{I}$ is an identity matrix. $\hat{\mathbf{s}}$, the estimation of the pulse response, is obtained by averaging the received samples of the first $N_{r}$ reference pulses:

$$
\hat{\mathbf{s}}=\frac{1}{N_{r}} \sum_{k^{\prime}=0}^{N_{r}-1} \mathbf{r}_{k^{\prime}}=\mathbf{s}+\frac{1}{N_{r}} \sum_{k^{\prime}=0}^{N_{r}-1} \mathbf{v}_{k^{\prime}}
$$

Correlating the received data pulse $\mathbf{r}_{k}$, where $k \geq N_{r}$, with $\hat{\mathbf{s}}^{H}$, the resulting input to the slicer is

$$
\begin{array}{r}
y_{k}^{\text {full }}=\hat{\mathbf{s}}^{H} \mathbf{r}_{k}=\left(\mathbf{s}^{H}+\frac{1}{N_{r}} \sum_{k^{\prime}=0}^{N_{r}-1} \mathbf{v}_{k^{\prime}}^{H}\right)\left(a_{k} \mathbf{s}+\mathbf{v}_{k}\right) \\
=a_{k} \mathbf{s}^{H} \mathbf{s}+\mathbf{s}^{H} \mathbf{v}_{k}+a_{k}\left(\frac{1}{N_{r}} \sum_{k^{\prime}=0}^{N_{r}-1} \mathbf{v}_{k^{\prime}}^{H}\right) \mathbf{s} \\
+\left(\frac{1}{N_{r}} \sum_{k^{\prime}=0}^{N_{r}-1} \mathbf{v}_{k}^{H} \mathbf{v}_{k}\right)
\end{array}
$$

where $H$ stands for conjugate transpose. In (26), the first term is the signal component and the remaining terms are the noise components. The second and third terms are clearly zero mean Gaussian noise. The fourth term, which is the noise-to-noise term, is also approximately Gaussian by the Central Limit Theorem, since the noise in different pulses is independent [3]. The variance of all the noise is

$$
\sigma_{\text {full }}^{2}=\sigma^{2} \mathbf{s}^{H} \mathbf{s}+\frac{1}{N_{r}} \sigma^{2} \mathbf{s}^{H} \mathbf{s}+\frac{N_{s} \sigma^{4}}{N_{r}}
$$

\section{B. Channelized Receiver}

Assuming $N_{s} / \gamma$ samples are collected at a rate of $1 / \gamma T_{e}(=$ $\left.1 / T_{s}\right)$ for each pulse, the ADC samples in the $m$ th subband of the $k$ th pulse is represented in vector form as

$$
\mathbf{x}_{k, m}=a_{k} \mathbf{s}_{m}+\mathbf{v}_{k, m}
$$

As in the full-band receiver, $\hat{\mathbf{s}}_{m}$, the estimation of the $m$ th subband pulse response, is obtained by averaging the received samples in the first $N_{r}$ reference pulses:

$$
\hat{\mathbf{s}}_{m}=\frac{1}{N_{r}} \sum_{k^{\prime}=0}^{N_{r}-1} \mathbf{x}_{k^{\prime}, m}=\mathbf{s}_{m}+\frac{1}{N_{r}} \sum_{k^{\prime}=0}^{N_{r}-1} \mathbf{v}_{k^{\prime}, m}
$$

Correlating with the samples in the $m$ th subband of the $k$ th data pulse, where $k \geq N_{r}$, then summing the output of all subbands,

$$
\begin{gathered}
y_{k}=\sum_{m=0}^{M-1} \hat{\mathbf{s}}_{m}^{H} \mathbf{x}_{k, m} \\
=\sum_{m=0}^{M-1}\left(\mathbf{s}_{m}^{H}+\frac{1}{N_{r}} \sum_{k^{\prime}=0}^{N_{r}-1} \mathbf{v}_{k^{\prime}, m}^{H}\right)\left(a_{k} \mathbf{s}_{m}+\mathbf{v}_{k, m}\right) \\
=\sum_{m=0}^{M-1}\left[a_{k} \mathbf{s}_{m}^{H} \mathbf{s}_{m}+\mathbf{s}_{m}^{H} \mathbf{v}_{k, m}+a^{k}\left(\frac{1}{N_{r}} \sum_{k^{\prime}=0}^{N_{r}-1} \mathbf{v}_{k^{\prime}, m}^{H}\right) \mathbf{s}_{m}\right. \\
\left.+\left(\frac{1}{N_{r}} \sum_{k^{\prime}=0}^{N_{r}-1} \mathbf{v}_{k^{\prime}, m}^{H}\right) \mathbf{v}_{k, m}\right]
\end{gathered}
$$

Similar to the full-band receiver, the input to the slicer consists of the signal term and three noise terms, the first two of which are Gaussian and the third that is approximately Gaussian. The variance of the additive noise is derived in the Appendix and is given by

$$
\sigma_{c h}^{2}=\sigma^{2} \mathbf{s}^{H} \mathbf{s}+\frac{1}{N_{r}} \sigma^{2} \mathbf{s}^{H} \mathbf{s}+\frac{M}{N_{r}} \operatorname{tr}\{\mathbf{R R}\}
$$

where $\mathbf{R}$ is the covariance matrix of $\mathbf{v}_{k, 0}$.

\section{Performance Comparison between Channelized and Full- Band Receiver}

The signal terms in (26) and (31) are the same due to the PR condition. Comparing (27) and (32), the variance of the additive noise for both the full-band and the channelized receivers are the same except for the last term. To determine $\operatorname{tr}\{\mathbf{R R}\}$, we first compute the sampled subband noise variance $\sigma_{s}^{2}$. Since $\mathbf{v}_{k, m}$ is obtained by passing a white noise $\mathbf{v}_{k}$ through a set of filters $\left\{H_{m}(\omega)\right\}$, which is the modulated version of a prototype filter, the noise variance of $\mathbf{v}_{k, m}$ in each subband is $\sigma_{s}^{2}$. The noise variance in each subband is 


$$
\sigma_{s}^{2}=E\left\{\mathbf{h}_{m}^{H} \mathbf{v}_{k} \mathbf{v}_{k}^{H} \mathbf{h}_{m}\right\}=\sigma^{2} \mathbf{h}_{m}^{H} \mathbf{h}_{m}=\frac{\gamma}{M} \sigma^{2}
$$

where $\mathbf{h}_{m}$ is the $m$ th analysis filter response, and the last equality, which is based on $\mathbf{h}_{m}^{H} \mathbf{h}_{m}=\gamma / M$, is obtained by applying the Parseval's theorem to the PR condition in (15).

If the receiver is maximally decimated, i.e., $\gamma=M$, and the analysis filters $\left\{H_{m}(\omega)\right\}$ are power complementary, $\mathbf{R}$ is a diagonal matrix. The last noise term in (32) then becomes

$$
\frac{M}{N_{r}} \operatorname{tr}\{\mathbf{R R}\}=\frac{M}{N_{r}} \frac{N_{s}}{\gamma}\left(\sigma_{s}^{2}\right)^{2}=\frac{\gamma}{M} \frac{N_{s} \sigma^{4}}{N_{r}}
$$

Since (34) is equal to the last noise term in (27), the channelized receiver achieves the same performance as the full-band receiver when maximally decimated, i.e., $\gamma=M$. When the channelized receiver is oversampled, i.e., $\gamma<M, \mathbf{R}$ is no longer a diagonal matrix. Since $\operatorname{tr}\{\mathbf{R R}\}$ is the sum of the power of all the elements in $\mathbf{R}$, both the diagonal and offdiagonal elements of $\mathbf{R}$ now contribute to $\operatorname{tr}\{\mathbf{R R}\}$. As the oversampling rate increases (or $\gamma / M$ decreases), the contribution to $\operatorname{tr}\{\mathbf{R R}\}$ by the diagonal elements of $\mathbf{R}$ decreases as can be seen from (34), but the contribution of the off-diagonal elements increases since the noise becomes more correlated.

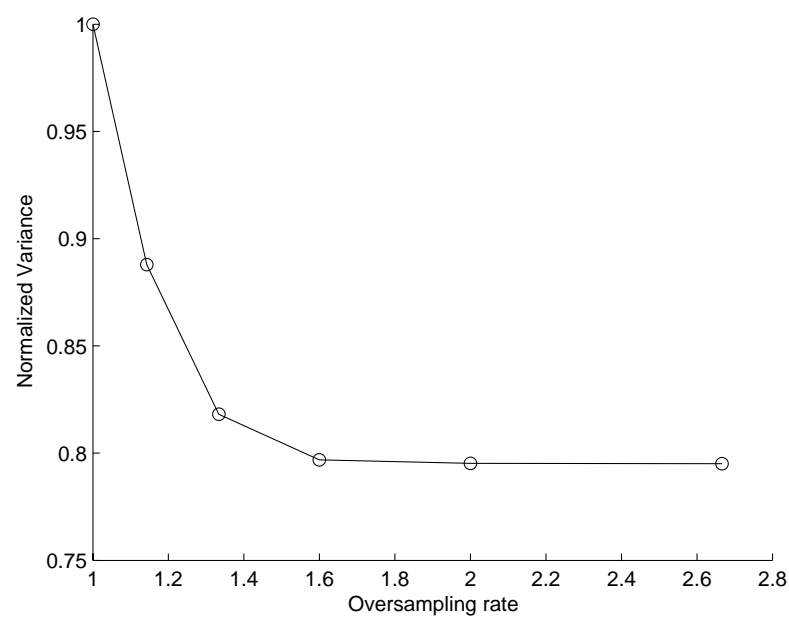

Fig. 6. Normalized variance vs. oversampling ratio.

An example of the net result is illustrated in Fig. 6, which plots the ratio of the last terms in (32) and (27) as a function of the oversampling ratio $M / \gamma$ with the filter bank obtained from [15]. As is clear from Fig. 6, the channelized receiver performs the same as the full-band receiver when maximally decimated. The oversampling channelized receiver, however, outperforms the full-band receiver with their gap in performance widening as the oversampling ratio increases. Beyond a certain oversampling ratio, no additional improvement in performance is observed.

Under typical operating conditions, the last terms in (32) and (27) contribute a large fraction to the total noise variance. For example, when $E_{b} / N_{0}=10 d B$, where $E_{b}=\mathbf{s}^{H} \mathbf{s}$ and $N_{0} / 2$ is the additive white Gaussian noise power density, and
$N_{s}=70$, which corresponds to a time interval of $20 \mathrm{~ns}$ when sampled at $1 / T_{e}=3.5 \mathrm{GHz}$, the third term in (27) contributes $64 \%$ of $\sigma_{\text {full }}^{2}$ for $N_{r}=1$ and $41 \%$ for $N_{r}=4$.

\section{AGC, NBI, AND COLORED NoISE}

\section{A. Detection with AGC}

In the previous sections, the effect of the ADC and the AGC were not considered. To fully utilize the dynamic range of the ADCs, an AGC is applied in each subband channel so that the percentage of the signal overflowing (or clipping) is kept within a certain percentage. This section describes how to account for the effect of the AGC when using the channelized receiver for detecting in UWB TR systems.

The $m$ th subband with an AGC is shown in Fig. 7. The AGC gain $A_{m}$ is set so that the power of $\mathbf{x}_{k, m}^{u}$, which is the signal plus noise at the input of the ADC, is kept constant. There is no pulse index $k$ in $A_{m}$, since the power of $\mathbf{x}_{k, m}^{u}$ is assumed constant within a transmission block. The downsampled subband signal is

$$
\mathbf{x}_{k, m}=a_{k} A_{m} \mathbf{s}_{m}+A_{m} \mathbf{v}_{k, m}
$$

The subband correlation signal is $A_{m} \mathbf{s}_{m}^{H}$, and the subband correlation value is

$$
y_{k, m}=a_{k} A_{m}^{2} \mathbf{s}_{m}^{H} \mathbf{s}_{m}+A_{m}^{2} \mathbf{s}_{m}^{H} \mathbf{v}_{k, m}
$$

For detection, the correlation value in each subband channel needs to be weighted by $1 / A_{m}^{2}$ to remove the effect of the AGC before summing. The resulting value is

$$
y_{k}=a_{k} \sum_{m=0}^{M-1} \alpha_{m} y_{k, m}
$$

where

$$
\alpha_{m}=K / A_{m}^{2}
$$

In (38), $K$ is some constant value, since detection depends on the ratio of $\alpha_{m}$ values.

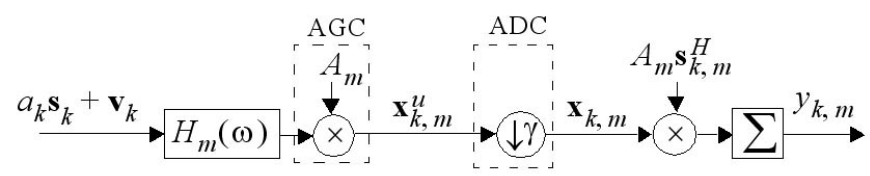

Fig. 7. Modeling of the effect of AGC in the $m$ th subband.

As the exact value of $A_{m}$ may be unknown in practice, $\alpha_{m}$ is estimated from the subband noise $A_{m} \mathbf{v}_{k, m}$. Since the noise variance of $\mathbf{v}_{k, m}$ is the same value $\sigma_{s}^{2}$ in all subbands, $\alpha_{m}$ can be expressed as

$$
\alpha_{m}=\frac{1}{E\left\{\left|A_{m} \mathbf{v}_{k, m}\right|^{2}\right\}}=\frac{1 / \sigma_{s}^{2}}{A_{m}^{2}}
$$

$E\left\{\left|A_{m} \mathbf{v}_{k, m}\right|^{2}\right\}$ can be obtained by estimating the noise variance of $\mathbf{x}_{k, m}$ in (35) based on the first $N_{r}$ reference pulses. The estimate of the noise variance of $\mathbf{x}_{k, m}$ is 


$$
\begin{aligned}
& \hat{\sigma}_{m}^{2}=\frac{1}{N_{r}-1} \sum_{k=0}^{N_{r}-1}\left(\mathbf{x}_{k, m}-\hat{\mathbf{s}}_{m}\right)^{H}\left(\mathbf{x}_{k, m}-\hat{\mathbf{s}}_{m}\right) \\
& =\frac{1}{N_{r}-1}\left[\sum_{k=0}^{N_{r}-1} \mathbf{x}_{k, m}^{H} \mathbf{x}_{k, m}-\frac{1}{N_{r}}\left|\sum_{k=0}^{N_{r}-1} \mathbf{x}_{k, m}\right|^{2}\right]
\end{aligned}
$$

\section{B. Detection with colored noise, NBI, and AGC}

As in [16], the NBI is modeled as a narrowband stochastic process. When colored noise or NBI is present, optimal detection is achieved by first whitening the noise then matched filtering the resulting signal. In channelized receivers, the whitening operation can be performed by appropriately scaling the correlation results in each subband before summing. To determine the scaling factors, we assume that the colored noise can be approximated by a white noise passing through weighted subband analysis filters as shown in Fig. 8(a). Denoting the weighting in the $m$ th subband as $B_{m}$, the $m$ th subband can be modeled as shown in Fig. 8(b). Note that in this model, the input pulse to the $m$ th subband channel is $\mathbf{s} / B_{m}$ instead of $\mathbf{s}$. The downsampled subband signal is

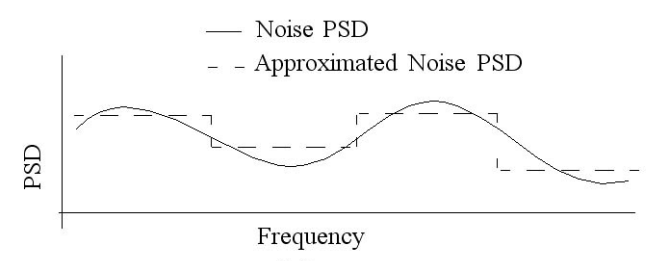

(a)

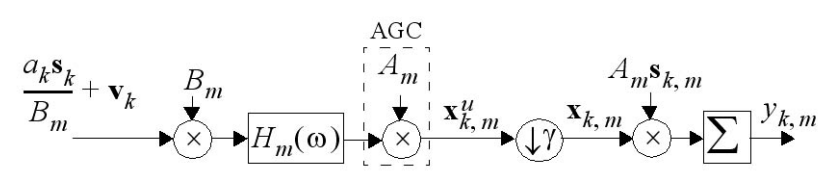

(b)

Fig. 8. (a) Approximation of colored noise using weighted subbands; (b) modeling the effect of colored noise in the $m$ th subband.

$$
\mathbf{x}_{k, m}=a_{k} A_{m} \mathbf{s}_{m}+A_{m} B_{m} \mathbf{v}_{k, m}
$$

Detection is then achieved by first scaling $\mathbf{x}_{k, m}$ with $1 / A_{m} B_{m}$ to whiten the noise and then correlating the result with $\mathbf{s}_{m}^{H} / B_{m}$ before combining.

The same result can be obtained by first correlating $\mathbf{x}_{k, m}$ with $A_{m} \mathbf{s}_{m}^{H}$ (or its estimate in practice) to obtain

$$
y_{k, m}=A_{m}^{2} \mathbf{s}_{m}^{H} \mathbf{s}_{m}+A_{m}^{2} B_{m}^{2}\left(\mathbf{s}_{m}^{H} / B_{m}\right) \mathbf{v}_{k, m}
$$

then scaling each subband after correlation by

$$
\alpha_{m}=\frac{K}{A_{m}^{2} B_{m}^{2}}
$$

As in the previous subsection, the estimate of $\alpha_{m}$ in (44) can be obtained by estimating the variance of $\mathbf{x}_{k, m}$ using (41).

\section{Simulation Results}

The transmitted monocycle is the second derivative of a gaussian pulse, i.e.,

$$
p(t)=\left[1-4 \pi\left(\frac{t}{t_{n}}\right)^{2}\right] \exp \left[-2 \pi\left(\frac{t}{t_{n}}\right)^{2}\right]
$$

where $t_{n}=1 \mathrm{~ns}$, which corresponds to a pulse width of approximately $2 \mathrm{~ns}$. The received noise free signal pulse is the superposition of monocycles with different delays and amplitudes. The multipath model used is the CM1 channel model recommended by the IEEE P802.15-02/368r5-SG3a. The effective sampling frequency is $1 / T_{e}=3.5 \mathrm{GHz}$. Assuming $M_{s}=4$ and $\gamma=5$, the ADC sampling rate is $7 / 5$ times the critically sampling rate. The lowpass filter $G(\Omega)$ in the analysis filter bank is a fourth order Butterworth filter, and its $3 \mathrm{~dB}$ frequency is at $1 /\left(12 T_{e}\right) . W(\omega)$ is obtained from [17]. The $N_{s}$ samples collected per pulse correspond to a time interval of 20ns, which represents collecting about $90 \%$ of the signal energy on average.

Fig. 9 shows the bit error rate (BER) of a UWB TR system assuming no knowledge of the signal pulse and the analysis filters. The first one or four reference pulses in a block of 100 pulses is used to estimate $s_{m}^{*}[l]$. This estimate is then used to detect the data pulses in the transmission block. The performance of an ideal matched filter receiver with perfect knowledge of the received signal pulse is shown as the lower bound. A full band receiver, which samples the UWB signal with a single ADC at the effective sampling rate and estimates the signal pulse by averaging the reference pulses, is used for comparison. As the number of reference pulses increases, the performance of both receivers approach that of the ideal receiver. The slight improvement in performance of the channelized receiver compared to the full-band receiver can be attributed in large part to the reduction in the noise-tonoise term (i.e., the last term in (32)) as described in Section IV.

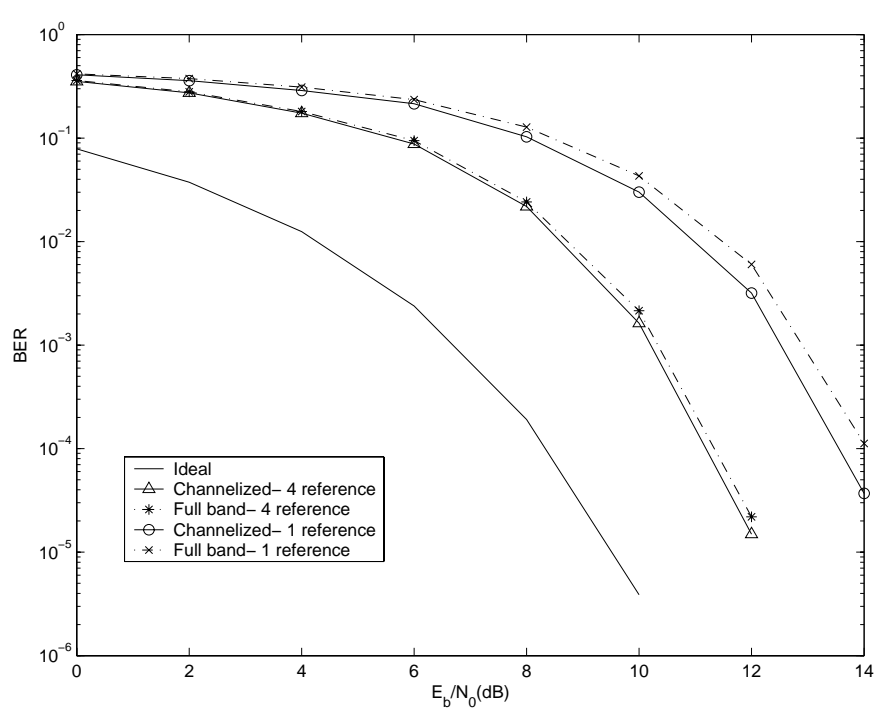

Fig. 9. BER vs. Eb/No for 1 and 4 reference pulses. 
The NBI is measured in terms of the signal-to-interference ratio (SIR), which is defined as the ratio of the received pulse energy of a single pulse $E_{b}$ to the interference power. The NBI is generated by passing a white noise through a sharp bandpass filter whose bandwidth is 0.05 of half the effective sampling frequency. In Fig. 10 and Fig. 11, we assume that four reference pulses are employed and the NBI center frequency is $0.57 f_{e} / 2$, which correspond to the frequency with the strongest signal power.

In Fig. 10, the BER is plotted as a function of $E_{b} / N_{0}$ for both the channelized and the full-band receivers when 1-bit, 3-bit, and infinite-bit ADCs are employed and the $S I R=$ $-5 d B$. As is clear from Fig. 10, the full-band receiver ceases to function regardless of the number of ADC bits. In contrast, the channelized receiver performs well at reasonable $E_{b} / N_{0}$ values as it is able to isolate the effect of the NBI. A 3-bit ADC seems sufficient to achieve performance comparable to a channelized receiver with infinite ADC bits.

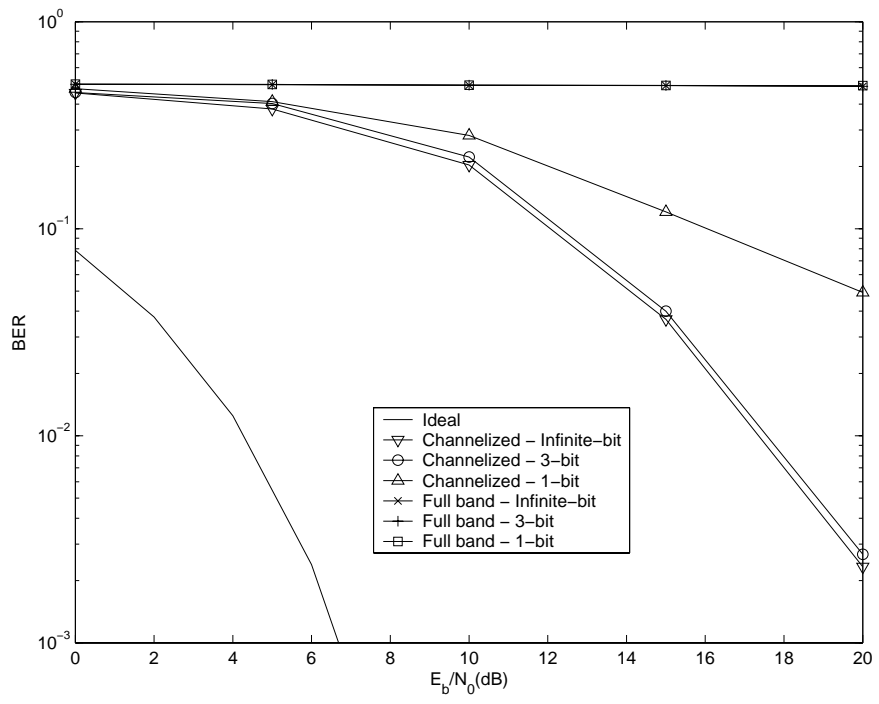

Fig. 10. BER vs. Eb/No for 1-bit, 3-bit and infinite-bit ADCs with $\mathrm{SIR}=-5 \mathrm{~dB}$ and 4 reference pulses.

Fig. 11 shows the BER for 1-bit, 3-bit, and infinite-bit ADCs as a function of SIR when $E_{b} / N_{0}=15 d B$. For the same BER, the chanelized receiver can tolerate significantly stronger NBI than the full-band receiver. For example, to achieve BER $=0.04$, a channelized receiver with 3-bit ADCs can tolerate almost $25 \mathrm{~dB}$ more interference than a full-band receiver with infinite-bit ADC. In the channelized receiver, little additional improvement in performance is obtained by increasing the ADC resolution beyond 3-bits. Thus, in UWB TR systems, the oversampled channelized receiver with only 3-bit ADC seems sufficient.

\section{CONCLUSIONS}

An oversampled channelized receiver for UWB TR systems is proposed. By combining the PR synthesis filters and the matched filter, detection is achieved by estimating on a persubband basis the combined responses of the channelizer and the propagation channel. The proposed receiver is shown to

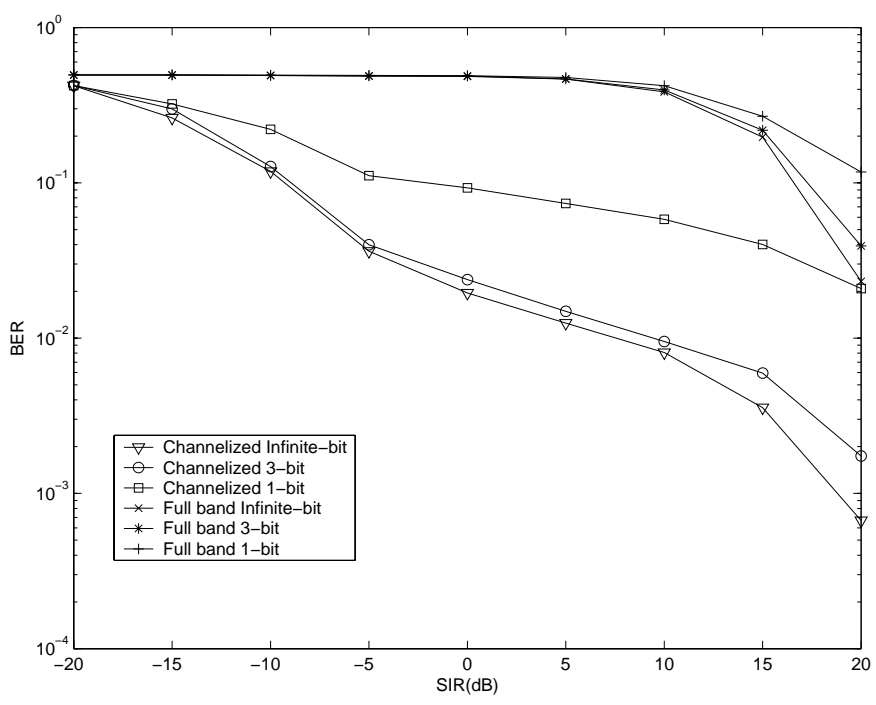

Fig. 11. BER vs. SIR with $E_{b} / N_{0}=15 \mathrm{~dB}$ and 4 reference pulses.

outperform the full-band receiver when the input noise is white. The oversampled channelized receiver is investigated in the presence AGC, ADC and NBI. If a strong narrowband interferer is present, the proposed receiver significantly outperforms the full band receiver because of its ability to isolate the effect of the NBI. Our results suggest that in TR UWB systems, the proposed receiver with ADC resolution of only 3 bits is enough to achieve performance comparable to an infinite bit channelized receiver even in the presence of large NBI.

\section{APPENDIX}

The channelized receiver noise power at the slicer input given in (32) is derived. Since the filter bank satisfies the PR condition, the variance of the first noise term is

$$
\begin{array}{r}
\operatorname{var}\left(\sum_{m=0}^{M-1} \mathbf{s}_{m}^{H} \mathbf{v}_{k, m}\right)=\operatorname{var}\left(\mathbf{s}^{H} \mathbf{v}_{k}\right) \\
=E\left\{\mathbf{s}^{H} \mathbf{v}_{k} \mathbf{v}_{k}^{H} \mathbf{s}\right\}=\sigma^{2} \mathbf{s}^{H} \mathbf{s}
\end{array}
$$

The variance of the second noise term is

$$
\begin{aligned}
& \operatorname{var}\left(\left(\frac{1}{N_{r}} \sum_{k^{\prime}=0}^{N_{r}-1} \mathbf{v}_{k^{\prime}, m}^{H}\right) \mathbf{s}_{m}\right) \\
= & E\left\{\left(\sum_{m_{1}=0}^{M-1} \mathbf{s}_{m_{1}}^{H}\left(\frac{1}{N_{r}} \sum_{k_{1}=0}^{N_{r}-1} \mathbf{v}_{k_{1}, m}\right)\right)\right. \\
& \left.\left(\sum_{m_{2}=0}^{M-1}\left(\frac{1}{N_{r}} \sum_{k_{2}=0}^{N_{r}-1} \mathbf{v}_{k_{2}, m_{2}}^{H}\right) \mathbf{s}_{m_{2}}\right)\right\} \\
= & \sum_{m_{1}=0}^{M-1} \sum_{m_{2}=0}^{M-1} \mathbf{s}_{m_{1}}^{H} E\left\{\frac{1}{N_{r}^{2}} \sum_{k_{1}=0}^{N_{r}-1} \sum_{k_{2}=0}^{N_{r}-1} \mathbf{v}_{k_{1}, m_{1}} \mathbf{v}_{k_{2}, m_{2}}^{H}\right\} \mathbf{s}_{m_{2}} \\
= & \frac{1}{N_{r}} \sum_{m_{1}=0}^{M-1} \sum_{m_{2}=0}^{M-1} \mathbf{s}_{m_{1}}^{H} E\left\{\mathbf{v}_{k, m_{1}} \mathbf{v}_{k, m_{2}}^{H}\right\} \mathbf{s}_{m_{2}}
\end{aligned}
$$




$$
\begin{aligned}
& =\frac{1}{N_{r}} \operatorname{var}\left(\sum_{m=0}^{M-1} \mathbf{s}_{m}^{H} \mathbf{v}_{k, m}\right) \\
& =\frac{1}{N_{r}} \sigma^{2} \mathbf{s}^{H} \mathbf{s}
\end{aligned}
$$

The second line from the bottom is due to the independence of noise between different pulses. The last line comes from (46).

The variance of the third noise term is

$$
\begin{array}{r}
\operatorname{var}\left(\sum_{m=0}^{M-1}\left(\frac{1}{N_{r}} \sum_{k^{\prime}=0}^{N_{r}-1} \mathbf{v}_{k^{\prime}, m}^{H}\right) \mathbf{v}_{k, m}\right) \\
=E_{k}\left\{\frac{1}{N_{r}} \sum_{m_{1}=0}^{M-1} \sum_{m_{2}=0}^{M-1} \mathbf{v}_{k, m_{1}}^{H} E_{k^{\prime}}\left\{\mathbf{v}_{k^{\prime}, m_{1}} \mathbf{v}_{k^{\prime}, m_{2}}^{H}\right\} \mathbf{v}_{k, m_{2}}\right\}
\end{array}
$$

where $E_{k}\{\}$ and $E_{k^{\prime}}\{\}$ represent taking the expectation of the $k$ th and $k^{\prime}$ th pulses, respectively. Evaluating $E_{k^{\prime}}\{\}$ at the cross of the $n_{1}$ th row and the $n_{2}$ th column.

$$
\begin{aligned}
& E_{k^{\prime}}\left\{v_{k^{\prime}, m_{1}}\left[n_{1}\right] v_{k^{\prime}, m_{2}}^{*}\left[n_{2}\right]\right\} \\
= & E\left\{\left(\sum_{n_{1}^{\prime}} v_{k^{\prime}}\left[n_{1}^{\prime}\right] h_{m_{1}}\left[\gamma n_{1}-n_{1}^{\prime}\right]\right)\right. \\
& \left.\left(\sum_{n_{2}^{\prime}} v_{k^{\prime}}\left[n_{2}^{\prime}\right] h_{m_{2}}^{*}\left[\gamma n_{2}-n_{2}^{\prime}\right]\right)\right\} \\
= & \sigma^{2} \sum_{n_{1}^{\prime}} h_{m_{1}}\left[\gamma n_{1}-n_{1}^{\prime}\right] h_{m_{2}}^{*}\left[\gamma n_{2}-n_{1}^{\prime}\right] \\
= & \sigma^{2} R_{m_{1}, m_{2}}\left[n_{1}, n_{2}\right] \\
\approx & \left.\sigma^{2} R_{m_{1}} n_{1}, n_{2}\right] \delta\left[m_{1}-m_{2}\right] \\
= & \sigma^{2} R\left[n_{1}, n_{2}\right] e^{j 2 \pi m_{1} \gamma\left(n_{1}-n_{2}\right) / M} \delta\left[m_{1}-m_{2}\right]
\end{aligned}
$$

The approximation in (49) is valid because the cross correlation $R_{m_{1}, m_{2}}\left[n_{1}, n_{2}\right]$ can be made approximately zero by minimizing the spectrum overlap between subbands using sharp filters. Although sharp filters are assumed to simplify the analysis, practical fourth-order Butterworth filters are used in the simulation results presented in Section VI. The last equality in (49) is obtained by making use of relationship between the $m$ th filter response $h_{m}[n]$ and the zeroth subband filter response $h[n]$ by $h_{m}[n]=h[n] e^{j 2 \pi n / M}$.

Put $R_{m}\left[n_{1}, n_{2}\right]$ to a matrix $\mathbf{R}_{m}$, which is the covariance matrix of subband noise $\mathbf{v}_{k, m}$. (48) then becomes

$$
\begin{aligned}
& \frac{1}{N_{r}} \sum_{m=0}^{M-1} E\left\{\mathbf{v}_{k, m}^{H} \mathbf{R}_{m} \mathbf{v}_{k, m}\right\} \\
= & \operatorname{tr}\left\{\frac{1}{N_{r}} \sum_{m=0}^{M-1} E\left\{v_{k, m}^{H} \mathbf{R}_{m} \mathbf{v}_{k, m}\right\}\right. \\
= & \frac{1}{N_{r}} \sum_{m=0}^{M-1} E\left\{\operatorname{tr}\left\{\mathbf{R}_{m} \mathbf{v}_{k, m} \mathbf{v}_{k, m}^{H}\right\}\right\} \\
= & \frac{1}{N_{r}} \sum_{m=0}^{M-1} \operatorname{tr}\left\{\mathbf{R}_{m} E\left\{\mathbf{v}_{k, m} \mathbf{v}_{k, m}^{H}\right\}\right\}
\end{aligned}
$$

$$
\begin{aligned}
& =\frac{1}{N_{r}} \sum_{m=0}^{M-1} \operatorname{tr}\left\{\mathbf{R}_{m} \mathbf{R}_{m}\right\} \\
& =\frac{1}{N_{r}} \sum_{m=0}^{M-1} \operatorname{tr}\{\mathbf{R R}\}
\end{aligned}
$$

where $\mathbf{R}=\mathbf{R}_{\mathbf{0}}$. Adding all three variances together,

$$
\sigma_{c h}^{2}=\sigma^{2} \mathbf{s}^{H} \mathbf{s}+\frac{1}{N_{r}} \sigma^{2} \mathbf{s}^{H} \mathbf{s}+\frac{M}{N_{r}} \operatorname{tr}\{\mathbf{R R}\}
$$

\section{REFERENCES}

[1] R. Hoctor and H. Tomlison, "An Overview of Delay-Hopped, Transmitted-Reference RF Communications," GE Research and Development Center, Tech. Rep. 2001CRD198, 2002.

[2] Y. Chao and R. Scholtz, "Optimal and Suboptimal Receiver for Ultra-Wideband Transmitted Reference Systems," IEEE GLOBECOM, pp. 759-763, Dec. 2003.

[3] J. Choi and W. Stark, "Performance of Ultra-Wideband Communications with Suboptimal Receivers in Multipath Channels," IEEE Journal on Selected Areas in Communications, vol. 20, no. 9, pp. 1754-1766, Dec. 2002.

[4] I. O'Donnell, S. Chen, S. Wang, and R. Brodersen, "An Integrated, Low Power, Ultra-Wideband Transceiver Architecture for Low-Rate, Indoor Wireless Systems," IEEE CAS Workshop on Wireless Communications and Networking, Sept. 2002.

[5] R. Blazquez, P. Newaskar, F. Lee, and A. Chandrakasan, "A Baseband Processor for Pulsed Ultra-Wideband Signals," IEEE Custom Integrated Circuits Conference, pp. 587-590, Oct. 2004.

[6] W. Namgoong, "A Channelized Digital Ultra-Wideband Receiver," IEEE Trans. Wireless Comm., vol. 2, pp. 502-510, May 2003.

[7] S. R. Velazquez, T. Q. Nguyen and S. R. Broadstone, "Design of Hybrid Filter Banks for Analog/Digital Conversion," IEEE Trans. on Signal Processing, vol. 46, no. 4, Apr. 1998.

[8] P. Lowenborg, H. Johansson and L. Wanhammar, "Two-Channel Digital and Hybrid Analog/Digital Multirate Filter Banks With Very LowComplexity Analysis or Synthesis Filters," IEEE Trans. on Circuits and Systems II: Analog and Digital Signal Processing, vol. 50, no. 7, Jul. 2003.

[9] A. Gilloire and M. Vetterli, "Adaptive Filtering in Subbands with Critical Sampling: Analysis, Experiments, and Application to Acoustic Echo Cancellation," IEEE Trans. on Signal Proc., vol. 40, no. 8, Aug. 1992.

[10] L. Feng and W. Namgoong, "A frequency channelized adaptive wideband receiver for high-speed links," Signal Processing Systems, 2003. SIPS 2003 IEEE Workshop on, 27 - 29 Aug. 2003, pp. 24-28.

[11] P. L. De Leon II and D. M. Etter, "Experimental Results with Increased Bandwidth Analysis Filters in Oversampled, Subband Acoustic Echo Cancelers," IEEE Signal Processing Letters, vol. 2, no. 1, Jan. 1995.

[12] R.E. Crochire and L. R. Rabiner, "Multirate Digital Signal Processing," Prentice Hall, 1983.

[13] S. Franz and U. Mitra, "On Optimal Data Detection for UWB Transmitted Reference Systems," IEEE GLOBECOM, pp. 744-748, Dec. 2003.

[14] H. Meyr, M. Moeneclaey and S. A. Fehtel, "Digital Communication Receivers: Synchronization, Channel Estimation, and Signal Processing," John Wiley \& Sons, Inc. 1998.

[15] T. Karp and N. J. Fliege, "Modified DFT Filter Banks with Perfect Reconstruction," IEEE Trans. on Circuits and Systems 2, vol. 46, no. 11, Nov. 1999.

[16] H. V. Poor, "Active Interference Suppression in CDMA Overlay Systems," IEEE Journal on Selected Areas in Communications, vol. 19, no. 1, Jan. 2001.

[17] M. Harteneck, S. Weiss, and R. W. Stewart, "Design of Near Perfect Reconstruction Oversampled Filter Banks for Subband Adaptive Filters,“ IEEE Trans. on Circuits and Systems Part II: Analog and Digital Signal Processing, vol. 46, no. 8, pp. 1081-1086, Aug. 1999. 


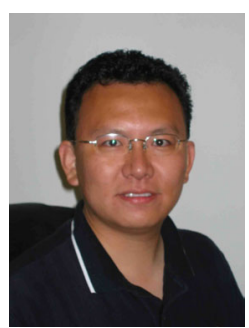

Lei Feng received the B.S. and M.S. degrees in electrical engineering from Peking University, Beijing, in 1997 and 2000, respectively. He is currently working toward the Ph.D degree in electrical engineering at the University of Southern California, Los Angeles, CA. His doctoral research focuses on the design of wideband communication transceivers for wireless and wireline applications.

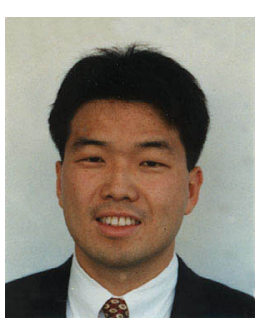

Won Namgoong received the BS degree in Electrical Engineering and Computer Science from the University of California at Berkeley in 1993, and the MS and Ph.D. degrees in Electrical Engineering from Stanford University in 1995 and 1999, respectively. In 1999, he joined the faculty of the Electrical Engineering Department at the University of Southern California, where he is an Assistant Professor. His current research areas include wireless/wireline communication systems, signal processing systems, RF circuits, and low-power/high-speed circuits. In 2002, he received the National Science Foundation (NSF) CAREER Award. He currently serves as the Associate Editor of IEEE Transactions on Circuits and Systems I - Regular Papers. 